\title{
Coordinative alignment of molecules in chiral metal-organic frameworks
}

Eugene Kapustin $^{1}$, Seungkyu Lee ${ }^{1}$, Omar Yaghi ${ }^{1}$

${ }^{1}$ University Of California Berkeley, Berkeley, United States

E-mail: kapustin@berkeley.edu

A chiral metal-organic framework, MOF-520, was used to coordinatively bind and align molecules of varying size, complexity, and functionality. The reduced motional degrees of freedom obtained with this coordinative alignment method allowed the structures of molecules to be determined by single-crystal $x$-ray diffraction techniques. The chirality of the MOF backbone also served as a reference in the structure solution for an unambiguous assignment of the absolute configuration of bound molecules. Sixteen molecules representing four common functional groups (primary alcohol, phenol, vicinal diol, and carboxylic acid), ranging in complexity from methanol to plant hormones (gibberellins, containing eight stereocenters), were crystallized and had their precise structure determined. We distinguished single and double bonds in gibberellins, and we enantioselectively crystallized racemic jasmonic acid, whose absolute configuration had only been inferred from derivatives.

Lee, S. et al. (2016). Science. 353, 808-811.
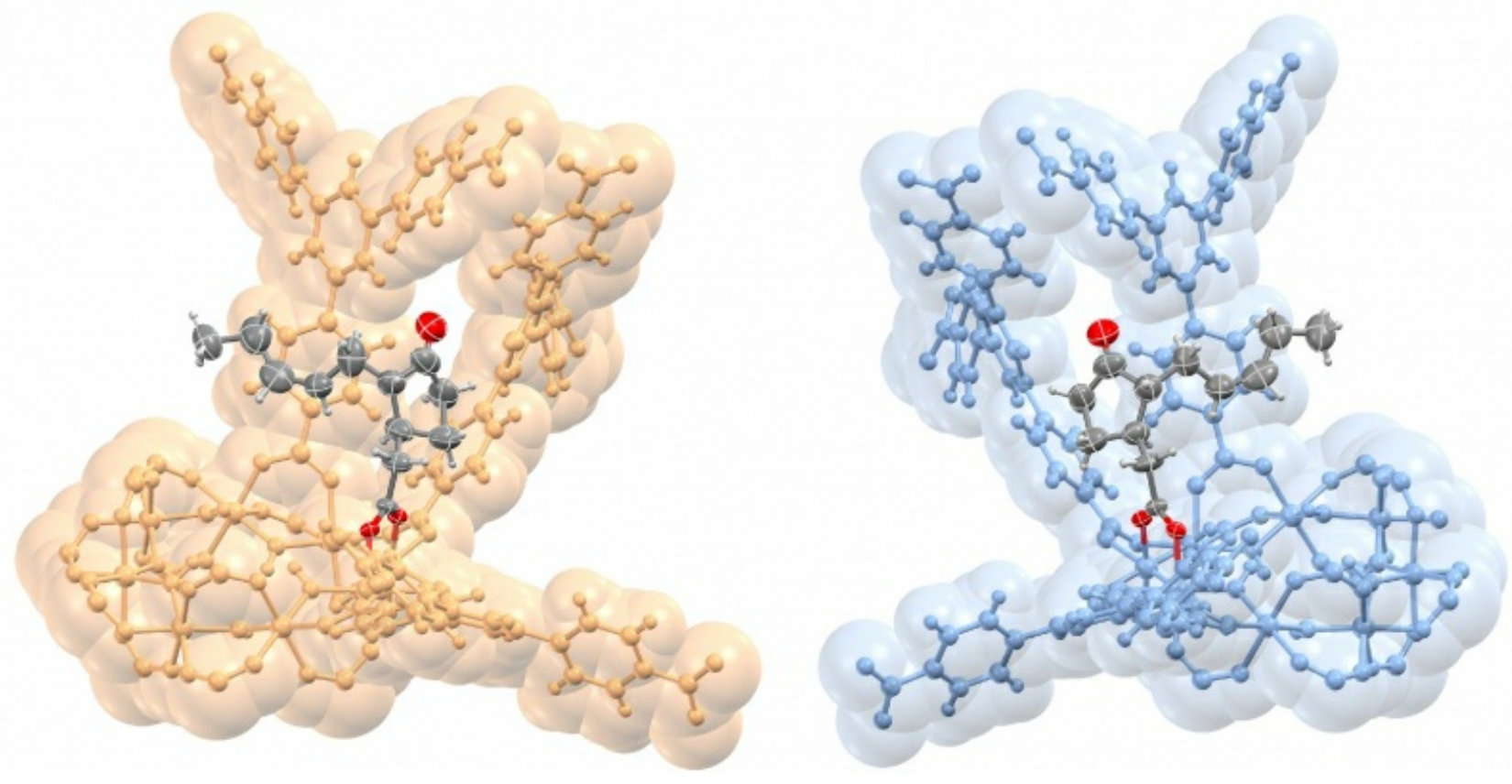

Keywords: metal-organic frameworks, absolute configuration, crystallization 